\title{
Effective Orifice Area
}

National Cancer Institute

\section{Source}

National Cancer Institute. Effective Orifice Area. NCI Thesaurus. Code C147149.

The minimal cross-sectional area of the flow jet downstream of a cardiac valve. 